\title{
REACCIÓN EMOCIONAL ANTE LA EXPOSICIÓN INVOLUNTARIA A CIBERSEXO EN ADOLESCENTES: FACTORES MODULADORES
}

\author{
Beatriz Gil-Juliá \\ Departamento de Personalidad, Evaluación y Tratamientos Psicológicos \\ Universitat de València (Spain) \\ beatriz.gil@uv.es \\ Jesús Castro-Calvo \\ Departamento de Personalidad, Evaluación y Tratamientos Psicológicos \\ Universitat de València (Spain) \\ Naiara Martínez-Gómez \\ Departamento de Psicología Básica, Clínica y Psicobiología \\ Universitat Jaume I, Castellón (Spain) \\ Verónica Cervigón-Carrasco \\ Departamento de Psicología Básica, Clínica y Psicobiología \\ Universitat Jaume I, Castellón (Spain) \\ María Dolores Gil-Llario \\ Departamento de Psicología Evolutiva y de la Educación \\ Universitat de València (Spain)
}

Fecha de Recepción: 19 Febrero 2019

Fecha de Admisión: 30 Abril 2019

\section{RESUMEN}

Introducción: Los avances realizados durante los últimos años en el ámbito de las tecnologías de la información y comunicación han favorecido un uso generalizado de internet. La población adolescente utiliza Internet de forma natural y habitual para comunicarse, entretenerse y realizar tareas escolares. Si bien son múltiples los beneficios derivados del uso de internet, es necesario considerar que no está exento de riesgos como puede ser la exposición involuntaria (El) a material sexual. Dadas las consecuencias que dicha exposición puede tener (sobre todo en edades tempranas), nuestro objetivo ha sido analizar las principales reacciones a nivel emocional durante la El a material sexual, explorando posibles diferencias en función del género, la edad de la primera El y el haber utilizado material pornográfico previamente. Método: 284 adolescentes (47,9\% chicos; $52,1 \%$ chicas) de entre 13-17 años (Media=14,8; DT=0,98), cumplimentaron un cuestionario ad hoc para la evaluación de diferentes dimensiones implicadas en la El a contenidos sexuales. Resultados: El $85,9 \%$ afirmaron haber sido expuestos involuntariamente a material sexual en internet, siendo la edad media de la primera exposición de 12,1 años $(\mathrm{DT}=1,66)$. Entre las principales reacciones emocionales, destacan la sorpresa $(53,3 \%)$, el asco $(37,6 \%)$, el shock $(21,9 \%)$ y la confusión $(21,9 \%)$. 


\section{REACCIÓN EMOCIONAL ANTE LA EXPOSICIÓN INVOLUNTARIA A CIBERSEXO EN ADOLESCENTES: FACTORES MODULADORES}

Asimismo, un 20,2\% experimentó interés o distracción, un 19,1\% vergüenza y un 19\% llegó a excitarse sexualmente. El análisis en función del género muestra diferencias significativas en sentir asco $\left(C_{i}{ }^{2}=39,37 ; p \leq 0,001\right)$, interés o distracción $\left(C h i^{2}=21,59 ; p \leq 0,001\right)$, excitación sexual $\left(C^{2} i^{2}=38,62\right.$; $p \leq 0,001)$, vergüenza (Chi $\left.{ }^{2}=15,86 ; p \leq 0,001\right)$, shock $\left(C_{i}{ }^{2}=12,59 ; p \leq 0,001\right)$ y confusión $\left(C^{2}{ }^{2}=4,21\right.$; $p=0,040$ ). En función de la edad, se obtienen correlaciones negativas significativas entre la edad de la primera El y experimentar interés o distracción $(R h 0=-0,202 ; p=0,006) 0$ sentir excitación sexual $(R h 0=-0,273 ; p \leq 0,001)$. En función de haber utilizado previamente material pornográfico, se observan diferencias en sentir asco $\left(\mathrm{Chi}^{2}=25,14 ; p \leq 0,001\right)$, interés o distracción $\left(C h{ }^{2}=26,19 ; p \leq 0,001\right)$, excitación sexual $\left(C h i^{2}=39,93 ; p \leq 0,001\right)$, vergüenza $\left(\mathrm{Chi}^{2}=13,60 ; p \leq 0,001\right)$ y shock $\left(\mathrm{Chi}^{2}=4,74\right.$; $\mathrm{p}=0,029)$. Conclusiones: Las reacciones emocionales de los adolescentes ante la El de contenido sexual online son diferentes en función de variables como el género, la edad de la primera El y el uso previo de material pornográfico. Conocer las variables que modulan dichas reacciones es fundamental para implementar programas de educación sexual ajustados a la realidad de nuestros adolescentes que permitan minimizar el impacto negativo que la El puede tener en su desarrollo psicosexual.

Palabras clave: exposición involuntaria; cibersexo; adolescentes; reacción emocional

\section{ABSTRACT}

Emotional reaction before involuntary exposure to cibersex in adolescents: modulator factors. Introduction: The advances in information and communication technologies during the last years have facilitated a widespread use of the Internet. Adolescents use the Internet as a natural and regular way to communicate, entertain and perform school tasks. Although many benefits may derive from the use of the Internet, it is necessary to consider that it is not exempt from risks such as involuntary exposure (IE) to sexual material. Given the consequences that such exposure can have (especially at early ages), our objective has been to analyze the main emotional reactions during the IE to sexual material, exploring possible differences according to gender, the age of the first IE and the previous use of pornography. Method. 284 adolescents (47.9\% boys; $52.1 \%$ girls) aged 13-17 years (mean=14.8; $S D=0.98$ ), completed an ad hoc questionnaire for the evaluation of different dimensions involved in IE to sexual contents. Results. $85.9 \%$ affirmed to have been exposed involuntarily to sexual material on the Internet, being the mean age of the first exhibition of 12.1 years $(S D=1.66)$. Among the main emotional reactions, surprise $(53.3 \%)$, disgust $(37.6 \%)$, shock $(21.9 \%)$ and confusion $(21.9 \%)$ stand out. Likewise, $20.2 \%$ experienced interest or distraction, $19.1 \%$ embarrassed and $19 \%$ became sexually aroused. Gender analysis shows significant differences in feeling disgust $\left(\mathrm{Chi}^{2}=39.37 ; p=0.000\right)$, interest or distraction $\left(\mathrm{Chi}^{2}=21.59 ; \mathrm{p}=0.000\right)$, sexual arousal $\left(\mathrm{Chi}^{2}=38.62 ; \mathrm{p}=0.000\right)$, shame $\left(\mathrm{Chi}^{2}=15.86 ; p=0.000\right)$, shock $\left(\mathrm{Chi}^{2}=12.59 ; p=0.000\right)$ and confusion $\left(\mathrm{Chi}^{2}=4.21 ; \mathrm{p}=0.040\right)$. Depending on the age, significant negative correlations are obtained between the age of the first IE and experiencing interest or distraction (Rho=-0.202; $p=0.006$ ) or feeling sexual arousal (Rho=-0.273; $p=0.000$ ). Based on having used pornographic material previously, there are differences in feelings of disgust $\left(\mathrm{Chi}^{2}=25.14 ; \mathrm{p}=0.000\right)$, interest or distraction (Chi $\left.{ }^{2}=26.19 ; p=0.000\right)$, sexual arousal $\left(\mathrm{Chi}^{2}=39.93 ; \mathrm{p}=0.000\right)$, shame $\left(\mathrm{Chi}^{2}=13.60\right.$; $p=0.000)$ and shock $\left(C_{i}^{2}=4.74 ; p=0.029\right)$. Conclusions: The emotional reactions of adolescents to online sexual content involuntarily are different depending on variables such as gender, the age of the first IE and the previous use of pornographic material. Knowing those variables that modulate these reactions is fundamental to implement sex education programs in keeping with the reality of our adolescents, which may minimize the negative impact caused by the IE on their psychosexual development.

Keywords: unwanted exposure; cybersex; teenagers; emotional reaction 


\section{INTRODUCCIÓN}

Los avances realizados durante los últimos años en el ámbito de las tecnologías de la información y comunicación (TIC) han favorecido un uso generalizado de internet especialmente entre los más jóvenes, quienes suelen estar mucho más familiarizados con su uso que los adultos. De hecho, en el año 2018 en España, el 86,1\% de la población de 16 a 74 años ha utilizado Internet en los últimos tres meses, porcentaje que asciende al 92,8\% cuando exploramos el uso de internet en niños de entre 10 y 15 años (INE, 2018). En esta misma línea, algunos estudios encuentran un mayor uso de las nuevas TIC, y con ellas de internet, en aquellos hogares en los que residen menores de edad (Observatorio de la Infancia en Andalucía, 2010). Asimismo, en todos los países europeos, incluido España, se registra un mayor porcentaje de acceso a Internet en los hogares con hijos/as a cargo (Eurostat, 2015).

No cabe duda de lo extendido que está el uso de internet habiéndose convertido en una herramienta prácticamente imprescindible que ha cambiado notablemente nuestra forma de comunicarnos e intercambiar información, ofreciendo múltiples y variadas ventajas (Mifsud, 2009). La población adolescente es una de las grandes beneficiarias de un uso adecuado de las TIC e internet, habiéndolo incorporado como un medio natural y habitual para comunicarse, entretenerse y realizar tareas escolares. Sin embargo, los múltiples beneficios junto con lo normalizado que está el uso de internet hacen que en numerosas ocasiones olvidemos que su uso no está exento de riesgos a los que son especialmente vulnerables los adolescentes. Algunos de estos peligros están relacionados con el fácil acceso a todo tipo de información, incluyendo por ejemplo contenidos violentos, xenófobos, pornográficos, etc., pudiendo además llegar a ser víctimas de solicitudes sexuales no deseadas, acoso 0 exposición involuntaria (El) a material sexual (Livingstone, Haddon, Görzig \& Ólafsson, 2011).

La El se entiende como "la exposición a imágenes de personas desnudas o manteniendo relaciones mientras una persona realiza una búsqueda online, navega por la red 0 abre su correo electrónico, siempre y cuando no busque voluntariamente ni espere ver material sexual" (p.337) (Mitchell, Finkelhor \& Wollak, 2003). Además, según algunos autores, esta El puede darse también en situaciones de interacción con otros usuarios teniendo en cuenta que no ha habido una elección previa sobre dicha exposición, es decir, la persona no ha elegido exponerse a dicho contenido (Bryant, 2009). En este sentido, globalmente se considerarían como El todas aquellas situaciones en las que una persona pueda recibir solicitudes sexuales no deseadas, sea acosada sexualmente a través de Internet 0 sea expuesta a contenidos sexuales explícitos de forma inesperada (Wollack, Mitchell \& Finkelhor, 2007).

Diversos estudios que se han centrado en analizar este fenómeno muestran cifras de prevalencia, cuanto menos significativas, que oscilan entre 23\% y 80\% (Ballester-Arnal, Giménez-García, GilLlario \& Castro-Calvo, 2016; Flood \& Hamilton, 2003; Mitchell et al., 2003; Mitchell, Wolak \& Finkelhor, 2007) siendo los adolescentes entre 15 y 17 años el grupo más afectado por esta El. Estos datos reflejan una realidad que parece ir en aumento además de cierta variabilidad entre contextos. Por el momento no es posible determinar cuál sería la tendencia general respecto a las cifras de EI dada la escasez de estudios llevados a cabo en diferentes países, si bien, a priori las políticas de control de contenidos en Internet podrían jugar un papel relevante en esta materia.

A partir de los datos disponibles, y sobre todo teniendo en cuenta que la población más afectada por esta exposición son los adolescentes, parece necesario plantearnos si esta El tiene impacto negativo sobre las personas expuestas. Si bien es un tema de entrada controvertido y que requiere de mayor grado de profundización, a partir de algunos estudios podemos afirmar que la El podría fomentar en los adolescentes valores y creencias erróneas en torno a la sexualidad, actitudes sexua- 
les excesivamente permisivas, promiscuidad sexual, edad de inicio en las relaciones sexuales muy temprana, un pobre autoconcepto, reacciones emocionales intensas (ansiedad, depresión, síntomas de TEPT), (Owens, Behun, Manning \& Reid, 2012) e incluso a más corto plazo, sensaciones de asco, disgusto, repulsión, vergüenza y shock (Aisbett, 2001).

Dadas las consecuencias que la El podría tener a nivel psicológico y también en el desarrollo sexual de los adolescentes, nuestro objetivo ha sido analizar las principales reacciones a nivel emocional durante la El a material sexual, explorando la existencia de posibles factores moduladores, como podrían ser el género, la edad de la primera El y haber utilizado material pornográfico previamente.

\section{MÉTODO}

\section{Participantes}

En este estudio han participado 284 adolescentes (47,9\% chicos; $52,1 \%$ chicas) de 3 institutos de Castellón de la Plana (España) con edades comprendidas entre los 13 y los 17 años (Media=14,8; $\mathrm{DT}=0,98)$. Los participantes del estudio estaban cursando 3ํ de la ESO (55\%) y $4^{\circ}$ de la ESO $(45 \%)$ en el momento de la evaluación.

\section{Instrumentos}

Para la evaluación de los participantes se ha utilizado un Cuestionario Ad-Hoc para explorar distintos aspectos de la El. Este instrumento está compuesto por 12 ítems con múltiples formatos de respuesta (escala tipo Likert, respuesta dicotómica, elección múltiple, etc.) que nos permiten explorar distintas dimensiones de la El, desde la frecuencia de exposición o la edad de la primera exposición hasta las reacciones a la El y sus consecuencias. Para este trabajo se seleccionaron los ítems que medían frecuencia de El, edad de la primera El y principales reacciones a nivel emocional durante la exposición involuntaria a material sexual.

\section{Procedimiento}

El instrumento de evaluación se aplicó en el marco de una investigación más amplia en el contexto escolar. Un psicólogo especialista en prevención fue el encargado de administrar los cuestionarios en clases de $3^{0}$ y $4^{0}$ de la ESO de 3 institutos de Castellón de la Plana. La aplicación del cuestionario (en formato lápiz y papel) fue grupal, si bien el encargado de la aplicación se aseguró de mantener el rigor exigido en toda investigación separando debidamente a los alumnos con el fin de mantener el anonimato y confidencialidad de las respuestas, así como solucionando cualquier duda que pudiera surgir.

\section{Análisis de los datos}

Los datos se analizaron mediante el paquete estadístico SPSS Versión 25.0. Para el cálculo de la prevalencia de la El se utilizó el total de participantes que componen la muestra. No obstante, para realizar el resto de análisis se eligieron únicamente aquellos participantes que afirmaron haber sido expuestos de forma involuntaria al menos en alguna ocasión a material sexual online. De esta manera, nos aseguramos la representatividad y posible generalización de los resultados obtenidos. Para el análisis de asociaciones entre variables se llevaron a cabo correlaciones de Spearman y para los análisis diferenciales la prueba estadística $\mathrm{Chi}^{2}$ por tratarse de variables categóricas.

\section{RESULTADOS}

El $85,9 \%$ de los adolescentes afirmaron haber sido expuestos involuntariamente a material sexual en internet al menos en alguna ocasión siendo la edad media de la primera exposición 12,1 
años $(\mathrm{DT}=1,66)$. Si bien es cierto que la mayoría de adolescentes que refieren haber sido expuestos contestaron que lo habían sido sólo "algunas veces" (35,7\%) o "casi nunca" $(43,9 \%)$, no podemos desestimar los datos que indican que un $4,1 \%$ y un $2,5 \%$ de los adolescentes señalan haber sido expuestos de forma involuntaria a estos contenidos muchas veces o muchísimas veces, respectivamente (Figura 1).

Figura 1. Frecuencia de exposición involuntaria en adolescentes (\%)

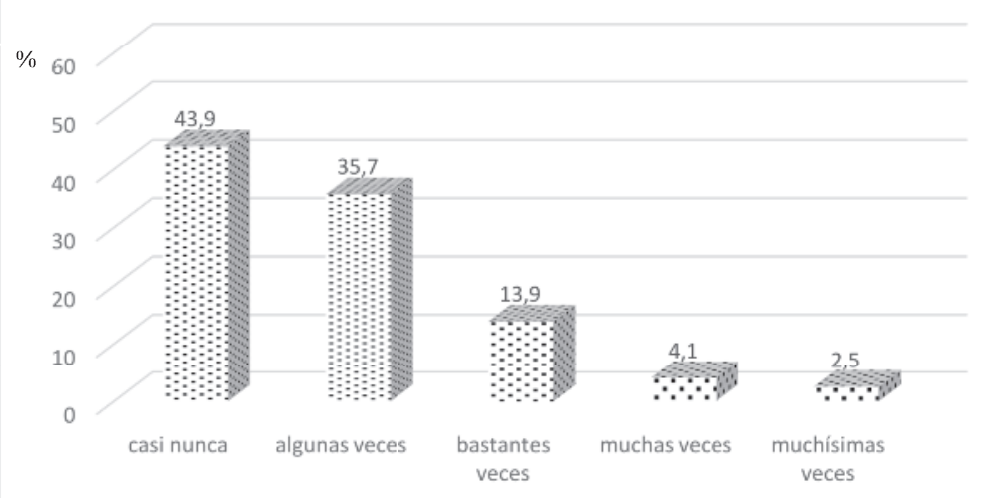

Entre las principales reacciones emocionales que presentaron los participantes durante la exposición destacan la sorpresa $(53,3 \%)$, el asco $(37,6 \%)$, el shock $(21,9 \%)$ y la confusión $(21,9 \%)$. Asimismo, un 20,2\% experimentó interés o distracción, un 19,1\% vergüenza y un $19 \%$ Ilegó a excitarse sexualmente. En cambio, las reacciones emocionales menos representadas fueron la tristeza $(0,8 \%)$ y la culpa $(1,2 \%)$ (Figura 2$)$.

Figura 2. Principales reacciones emocionales durante la EI

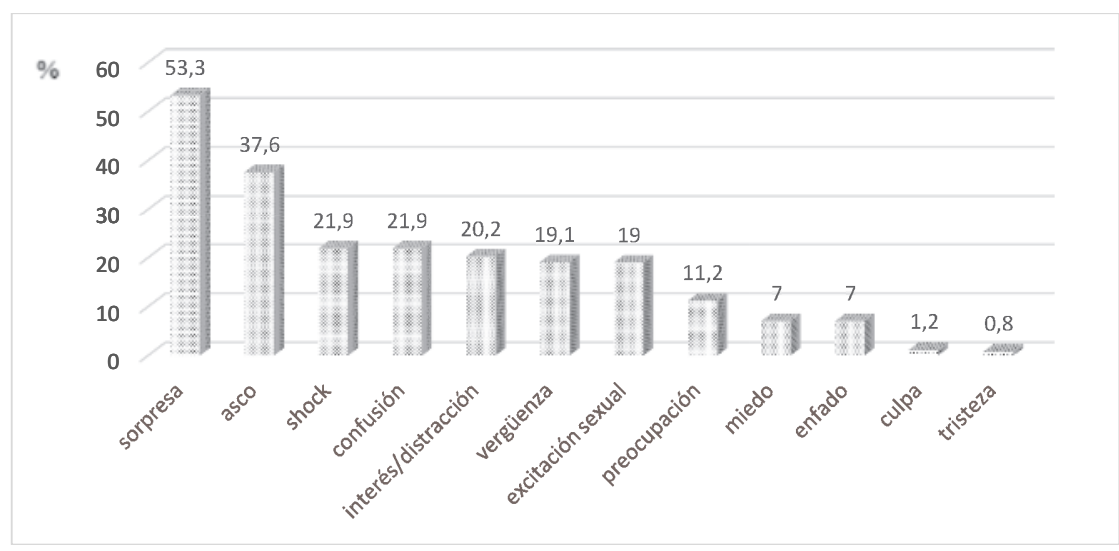


El análisis de los resultados en función del género muestra diferencias significativas entre chi$\cos$ y chicas en las reacciones emocionales experimentadas durante la El. En este sentido, las chicas refieren experimentar significativamente más asco $\left(\mathrm{Chi}^{2}=39,37 ; \mathrm{p} \leq 0,001\right)$, vergüenza $\left(C_{i}{ }^{2}=15,86 ; p \leq 0,001\right)$ y shock $\left(C^{2}{ }^{2}=12,59 ; p \leq 0,001\right)$ que los chicos. Por su parte, estos últimos, ante la El experimentan mayor interés o distracción (Chi $\left.{ }^{2}=21,59 ; p \leq 0,001\right)$, excitación sexual $\left(\mathrm{Chi}^{2}=38,62 ; p \leq 0,001\right)$ y confusión $\left(\mathrm{Chi}^{2}=4,21 ; \mathrm{p}=0,040\right)$ que las chicas (Tabla 1).

En función de la edad, se obtienen correlaciones negativas significativas entre la edad de la primera El y experimentar interés o distracción ( $R h 0=-0,20 ; p=0,006) 0$ sentir excitación sexual (Rho=$0,27 ; p \leq 0,001)$, es decir, estas reacciones emocionales han sido mayores cuanto más pronto fueron expuestos los adolescentes de forma involuntaria a contenido sexual online (Tabla 1).

El análisis de los resultados en función de haber utilizado previamente material pornográfico, muestra diferencias significativas en algunas de las reacciones emocionales experimentadas. En este sentido, los participantes que no han utilizado previamente material pornográfico experimentan significativamente más emociones que podríamos considerar negativas como el asco $\left(\mathrm{Chi}^{2}=25,14\right.$; $p \leq 0,001)$, la vergüenza $\left(C^{2} i^{2}=13,60 ; p \leq 0,001\right)$ y el shock $\left(C h i^{2}=4,74 ; p=0,029\right)$. En cambio, los adolescentes que han utilizado material pornográfico previamente muestran reacciones más positivas como un mayor interés o distracción $\left(C h i^{2}=26,19 ; \quad p \leq 0,001\right)$ así como excitación sexual $\left(\mathrm{Chi}^{2}=39,93 ; \mathrm{p} \leq 0,001\right)$ durante la El a este tipo de contenido (Tabla 1)

Tabla 1. Principales reacciones emocionales durante la El en función del género, edad 1aㅡ exposición y uso de pornografía

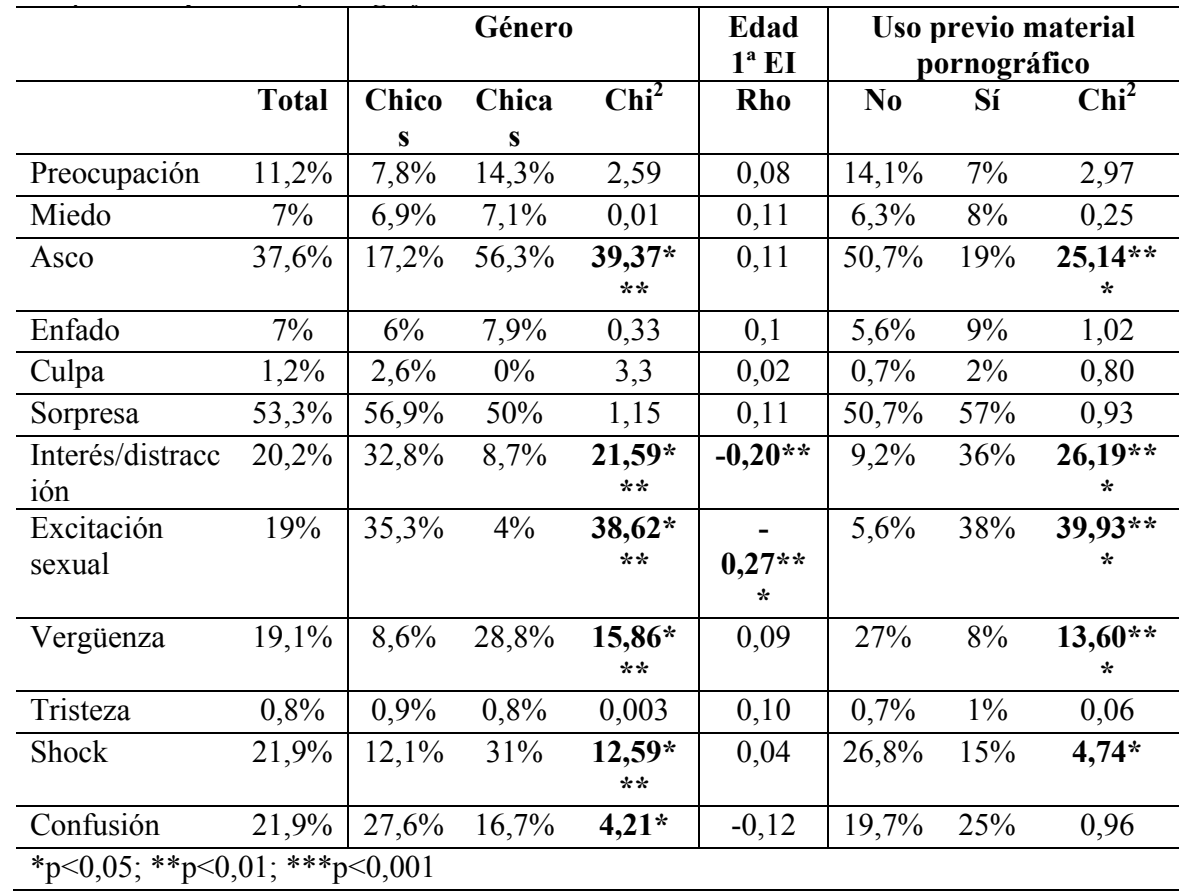




\section{DISCUSIÓN Y CONCLUSIONES}

Los resultados de este trabajo nos permiten conocer con más de detalle cuál es la realidad de la El en nuestro contexto y de manera particular profundizar en el análisis de las reacciones emocionales que puedan aparecer tras la citada exposición. En primer lugar, podemos afirmar que la mayoría de los escolares evaluados han sido expuestos de forma involuntaria a material sexual online. Estos resultados van en la línea de los obtenidos en algunos estudios previos que estiman cifras de prevalencia de El en torno al 80\% (Castro-Calvo, Gómez-Martínez, Gil-Juliá, Giménez-García \& Ballester-Arnal, 2015; Flood \& Hamilton, 2003), en contraposición con datos mucho más modestos obtenidos a partir de estudios realizados en contextos en los que parece haber un acceso más restrictivo a contenido ilegal online que el que podamos encontrar en países de Europa y/o Australia (Buljan-Flander, Cosic \& Profaca, 2009; Flood, 2007; Flood \& Hamilton, 2003).

En cuanto a las reacciones emocionales suscitadas a partir de la El, los datos muestran sobre todo la presencia de sorpresa, asco, shock, vergüenza y confusión, observándose por tanto en general un mayor predominio de lo que podríamos considerar como emociones más negativas. La literatura revisada ofrece cierta tendencia a experimentar emociones negativas ante la El, sobre todo en niños y adolescentes (Aisbett, 2001).

Un análisis más detallado sobre las reacciones emocionales que presentan los adolescentes tras la El indica que éstas pueden variar en función de una serie de variables como el género, la edad de la primera El y el uso previo de material pornográfico. En este sentido, las principales diferencias podemos entenderlas desde la valencia asociada a estas reacciones emocionales. Por un lado, las chicas suelen presentar reacciones más negativas sobre todo de asco, vergüenza y shock, mientras que en contraste son los chicos, quienes parecen responder además de con cierta confusión, con más interés y excitación sexual ante la El. Estos datos sobre mayor interés y excitación sexual, en principio pueden resultar un tanto sorprendentes puesto que nos estamos refiriendo a una exposición no voluntaria a material sexual y además a edades tempranas. Una posible explicación podríamos encontrarla en el hecho de que los chicos suelen presentar también una mayor disposición erotofílica (Paul, 2009) y una mayor predisposición y actitud positiva hacia el consumo de material pornográfico (Carroll et al., 2008). Asimismo, estas reacciones de interés, distracción o sentir excitación sexual han sido mayores cuanto más pronto fueron expuestos los adolescentes de forma involuntaria a contenido sexual online. Estos datos irían en la línea de lo que acabamos de exponer puesto que son los chicos quienes más tempranamente se exponen de forma involuntaria a cibersexo (Gil-Juliá, Castro-Calvo, Ruiz-Palomino, García-Barba \& Ballester-Arnal, 2018; Sabina, Wolak \& Finkelhor, 2008).

Finalmente, este estudio ofrece datos importantes sobre el papel modulador que el visionado previo de material pornográfico puede tener en las reacciones emocionales ante la El a dicho contenido. Los adolescentes que no han utilizado previamente material pornográfico experimentan más emociones que podríamos considerar negativas como el asco, la vergüenza y el shock. En cambio, aquellos que han utilizado material pornográfico con anterioridad muestran reacciones más positivas, como un mayor interés o distracción y excitación sexual durante la El. Estos datos están relacionados con los obtenidos en otros estudios que revelan menos reacciones de rechazo entre los sujetos que habitualmente han consumido cibersexo (Strasburger \& Wilson, 2002; Thornburgh \& Lin, 2002). En este sentido, la exposición voluntaria previa a cibersexo podría considerarse por tanto como un factor a tener en cuenta a la hora de amortiguar los efectos emocionales más negativos que pueden aparecer durante la El a cibersexo.

Este estudio ofrece datos relevantes sobre variables que pueden modular las reacciones emocionales durante la El a material sexual, las cuales deberían tenerse en consideración sobre todo de 
cara a implementar programas de educación afectivo-sexual y de prevención ajustados a la realidad de nuestros adolescentes, que minimicen el impacto negativo que la El puede tener en su desarrollo psicosexual.

De igual forma, es necesario seguir profundizando en el análisis de un tema que afecta a tantos menores habida cuenta de las repercusiones que puede tener, siendo necesario ampliar el estudio incluyendo más zonas de nuestra geografía e incluso otros países europeos que nos permitan confirmar y generalizar los hallazgos obtenidos.

\section{REFERENCIAS BIBLIOGRÁFICAS}

Aisbett, K. (2001). The Internet at Home: A report on Internet use in the home. Sydney: Australian Broadcasting Authority.

Ballester-Arnal, R., Giménez-García, C., Gil-Llario, M.D. \& Castro-Calvo, J. (2016). Cybersex in the "Net generation": Online sexual activities among Spanish adolescents. Computers in Human Behavior, 57, 261-266.

Buljan-Flander, G., Cosic, I. \& Profaca, B. (2009). Exposure of children to sexual content on the Internet in Croatia. Child Abuse \& Neglect, 33, 849-856.

Bryant, C. (2009). Adolescence, pornography and harm. Trends \& Issues in Crime and Criminal Justice,368, 1-6.

Carroll, J. S., Padilla-Walker, L. M., Nelson, L. J., Olson, C. D., McNamara C. \& Madsen, S. D. (2008). Generation XXX: Pornography Acceptance and Use Among Emerging Adults. Journal of Adolescent Research, 23, 6-30.

Castro-Calvo, J., Gómez-Martínez, S., Gil-Juliá, B., Giménez-García, C. \& Ballester-Arnal, R. (2015). Jóvenes y sexo en la red. Reacción ante la exposición involuntaria a material sexual. Agora Salut, 1,187-198.

Eurostat (2015). Being young in Europe today. Eurostat Statistical Books. Luxembourg: Publications Office of the European Union. Disponible: http://ec.europa.eu/eurostat/documents/3217494/6776245/KS-05-14-031-EN-N.pdf/18bee6f0-c181-457d-ba82-d77b314456b9.

Flood, M. (2007). Exposure to pornography among youth in Australia. Journal of Sociology, 43 (1), 45-60.

Flood, M. \& Hamilton, C. (2003). Youth and pornography in Australia: evidence on the extent of exposure and likely effects (informe de The Australia Institute $n . \stackrel{0}{52}$ ). Canberra: The Australia Institute.

Gil-Juliá, B., Castro-Calvo, J., Ruiz-Palomino, E., García-Barba, M., Ballester-Arnal, R. (2018). Consecuencias de la exposición involuntaria a material sexual en adolescentes. International Journal of Developmental and Educational Psychology, 1, 33-44.

Instituto Nacional de estadística, INE. (2018). Encuesta sobre Equipamiento y Uso de Tecnologías de la Información y Comunicación en los hogares 2018. Consultado el 18 de abril de 2019 en http://www.ine.es/dyngs/INEbase/es/operacion.htm?c=Estadistica_C\&cid=1254736176741\&m $\underline{\text { enu}}=u$ ultiDatos\&idp $=1254735976608$

Observatorio de la Infancia en Andalucía (2010). Encuesta sobre equipamientos y uso de tecnologías de la información y la comunicación en los hogares 2009. Consejería de Innovación, Ciencia y Empresa. Junta de Andalucía.

Paul, B. (2009). Predicting Internet Pornography Use and Arousal: The Role of Individual Difference Variables. Journal of Sex Research, 46 (4), 344-357.

Livingstone, S., Haddon, L., Görzig, A., \& Ólafsson, K. (2011). Risks and safety on the internet: The perspective of European children. Full Findings. LSE, London: EU Kids Online. 
Mifsud, E. (2009). Monográfico: Control parental-Uso de internet: riesgos y beneficios. Ministerio de Educación, Cultura y Deporte. Consultado el 20 de abril de 2019 en http://recursostic.educacion.es/observatorio/web/eu/software/software-general/909-monografico-controlparental?start=1

Mitchell, K. J., Finkelhor, D., \& Wollak, J. (2003). The exposure of youth to unwanted sexual material on the Internet: A national survey of risk, impact, and prevention. Youth \& Society, 34, 330358.

Mitchell, K. J., Wolak, J. \& Finkelhor, D. (2007). Trends in youth reports of sexual solicitations, harassment and unwanted exposure to pornography on the Internet. Journal of Adolescent Health, 40, 116-126.

Sabina, C., Wolak, J. \& Finkelhor, D. (2008) The nature and dynamics of internet pornography exposure for youth. CyberPsychology \& Behavior, 11(6), 1-3.

Owens, E. W., Behun, R. J, Manning, J. C. \& Reid, R. C. (2012). The Impact of Internet Pornography on Adolescents: A Review of the Research. Sexual Addiction \& Compulsivity, 19, 99-122.

Wollack, J., Mitchell, K., \& Finkelhor, D. (2007). Unwanted and Wanted Exposure to Online Pornography in a National Sample of Youth Internet Users. Pediatrics, 119(2), 247-257. 
